\title{
Recurrent Chronic Lymphocytic Leukemia/Small Lymphocytic Lymphoma
}

National Cancer Institute

\section{Source}

National Cancer Institute. Recurrent Chronic Lymphocytic Leukemia/Small Lymphocytic Lymphoma. NCI Thesaurus. Code C131873.

The reemergence of chronic lymphocytic leukemia/small lymphocytic lymphoma after a period of remission. 